\title{
Chronic Stress, Sense of Belonging, and Depression Among Survivors of Traumatic Brain Injury
}

\author{
Esther Bay, Bonnie M. Hagerty, Reg A. Williams, Ned Kirsch, Brenda Gillespie
}

\begin{abstract}
Purpose: To test whether chronic stress, interpersonal relatedness, and cognitive burden could explain depression after traumatic brain injury (TBI).

Design: A nonprobability sample of 75 mild-to-moderately injured TBI survivors and their significant others, were recruited from five TBI day-rehabilitation programs. All participants were within 2 years of the date of injury and were living in the community.

Methods: During face-to-face interviews, demographic information, and estimates of brain injury severity were obtained and participants completed a cognitive battery of tests of directed attention and short-term memory, responses to the Perceived Stress Scale, Interpersonal Relatedness Inventory, Sense of Belonging Instrument, Neurobehavioral Functioning Inventory, and Center for Epidemiological Studies Depression Scale;.

Findings: Chronic stress was significantly and positively related to post-TBI depression. Depression and postinjury sense of belonging were negatively related. Social support and results from the cognitive battery did not explain depression.

Conclusions: Postinjury chronic stress and sense of belonging were strong predictors of postinjury depression and are variables amenable to interventions by nurses in community health, neurological centers, or rehabilitation clinics. Future studies are needed to examine how these variables change over time during the recovery process.
\end{abstract}

Journal of Nursing Scholarship, 2002; 34:3, 221-226. @2002 Sigma Theta Tau International.

[Key words: chronic stress, sense of belonging, traumatic brain injury, depression]

$\mathrm{T}$ raumatic brain injury (TBI), a major public health concern throughout the world, is associated with chronic health changes and it affects $2 \%$ of the United States population or 5.3 million Americans. Significant neurobehavioral, mood, and cognitive changes, evident even after a seemingly mild injury, result in admission to community rehabilitation programs. The annual costs of acute care and rehabilitation for new cases of TBI are estimated to be \$9-\$10 billion (National Institutes of Health [NIH] Consensus Statement, 1998).

Of those recovering from TBI, $66 \%$ are men under age 35 , who are otherwise developmentally able to perform productive work and social roles (NIH Consensus Statement, 1998). Rehabilitation specialists' goals are to assist survivors and families to develop strategies for resuming these roles. Depression, identified as a major mood disorder after moderate and severe TBI (Hibbard, Uysal, Kepler, Bogdany, \& Silver, 1998; Satz et al., 1998) and problematic after mild brain injury (Busch \& Alpern, 1998; NIH Consensus Statement, 1998), complicates this process. Post-TBI depression is most likely underdiagnosed and implicated in rising rates of substance abuse and marital or work conflicts, common to this population.

The purpose of this cross-sectional and exploratory study was to test whether chronic stress, interpersonal relatedness, and cognitive burden, variables found to be associated with clinical depression, could help explain post-TBI depression.

\section{Background}

Depression is viewed as a constellation of depressive symptoms with specific focus on affective responses. These

\footnotetext{
Esther Bay, RN, PhD, APRN, BC, Lambda, Assistant Professor, Michigan State University, Dearborn; Bonnie M. Hagerty, RN, PhD, CS, Rho, Associate Professor of Nursing; Reg A. Williams, RN, PhD, CS, Rho, Professor of Nursing; Ned Kirsch, PhD, Assistant Professor and Director of Adult TBI Program; Brenda Gillespie, PhD, Assistant Professor and Co-director of the Center for Statistical Consulting and Research; all at the University of Michigan, Ann Arbor. Correspondence to Dr. Bay, 2106 Kratage Court, Commerce, MI 48382. E-mail: pdq@umich.edu

Accepted for publication March 25, 2002.
} 
symptoms could escalate to severe dysfunction, thereby increasing suicide potential (Ownsworth \& Oei, 1998). Depressive symptoms may represent the construct of demoralization, believed to accompany severe physical and psychological illness, and are associated with hopelessness, sadness, and low self-esteem (Breslau \& Davis, 1986). When compared to people with chronic medical conditions, those only with depressive symptoms were significantly more impaired in social functioning. An additive effect was reported when medically ill people also had depressive symptoms (Wells et al., 1989).

Methodological refinements are needed in post-TBI depression research with greater focus on explanatory models and interventions. An extensive review of current models of post-TBI depression, measurement indices, and methodological concerns is reported elsewhere (Bay, 2000), yet two longitudinal studies were focused on the 1st-year perspective of TBI recovery and depression (McCleary et al., 1998; Jorge et al., 1993) served as a guide in this study. In brief, when depressed and nondepressed survivors were compared during the first weeks of recovery, structural differences were found. Severity of injury, although related to depression during the first 6 months after injury, was not a significant explanatory variable at the 1 -year evaluation, and psychosocial variables persisted or worsened over time. These findings show that depression is not simply the result of a biological lesion or response to a catastrophic event but that it is functionally related to the time since injury and the effects of social connections and support. Thus, a more comprehensive perspective than severity alone is required.

\section{Postinjury Stress and Depressive Symptoms}

Consider this model case of chronic stress after TBI. A family of three is unexpectedly involved in a motor vehicle crash. The father sustains a spinal cord injury and is transported to a regional trauma center, the mother experiences a moderate brain injury, and their 10-year-old daughter appears unharmed, but later she is diagnosed with post traumatic stress disorder. The mother is treated in an outpatient facility and while undergoing rehabilitation for herself, must also learn how to care for her quadriplegic husband and the psychologically traumatized daughter. Financial obligations, workload responsibilities, and care for other family members are required. The mother experiences relentless and continuous stress over the ensuing 6 months and is treated for depression.

Can chronic stress account for depression after brain injury? Consistent evidence exists that chronic stressors associated with circumstances of intense duration increase vulnerability to clinical depression (Baum, Gatchel, \& Schaeffer, 1983; Breslau \& Davis, 1986; Brooks, 1999; Cohen, Kessler, \& Gordon, 1995). TBI theorists have proposed that stress and coping responses contribute to psychosocial and emotional difficulties after TBI (Kendall \& Terry, 1996; Martelli, Zasler, \& MacMillan, 1998; Montgomery, 1995; Moore \& Stambrook, 1995), yet the specificity and empirical adequacy of these models has yet to be determined.
For this investigation, chronic stress was defined as a pernicious state resulting from persistent demands of the environment or from an event or experience perceived as threatening or demanding because it exceeded the person's physical or psychological adaptive capacity and it precipitated a sequential chain of physical, emotional, and interpersonal difficulties. Chronic stress present after the injury is hypothesized to result in greater depressive symptoms.

\section{Interpersonal Relatedness and Depression}

These negative effects of stress can be alleviated by supportive relationships with others (Cohen \& Wills, 1985; Hagerty \& Williams, 1999; House, Landis, \& Umberson, 1988). Social support and sense of belonging, measures of interpersonal relatedness, are posited to attenuate the adverse effects of stress, thereby reducing depressive symptoms.

After TBI, survivors have reported notable declines in social networks (Kozloff, 1987; Oddy \& Humphrey, 1980; Thomsen, 1984), and those without confidants reported more emotional difficulties (Kinsella, Moran, Ford, \& Ponsford, 1988) and impairments in social functioning (Lezak, 1987). Yet, Jorge and associates (1993) were unable to establish the cause-effect relationship of social networks and depression in their longitudinal study, and Leach, Frank, Bouman, and Farmer (1994) reported that survivor satisfaction with social support was not related to levels of depression. Others have speculated that psychosocial variables were implicated (McCleary et al., 1998; Rosenthal, Christensen, \& Ross, 1998).

For this study, social support was defined as a person's perception that a person or group provides helping behaviors that result in emotional benefit or gains in tangible materials. These helping behaviors reduce the negative effect of chronic stress by interfering with the duration of exposure and disrupting the sequential chain of events that characterize chronic stress. Most likely, social support modifies the perception of the stress event and it interrupts the process of cascading negative events (Cohen \& Wills, 1985).

This study also was focused on the relationship between sense of belonging and post-TBI depression. This second measure of interpersonal relatedness is defined as valued fit and involvement with another, a group, an object, or the environment (Hagerty, Lynch-Sauer, Patusky, Bouwsema, \& Collier, 1992). It differs from social support, yet it has been linked to depression. In a path analysis of the predictive relationship of interpersonal variables and belonging on depression, sense of belonging helped to explain a significant amount of depression, and loneliness and depression were modeled as consequences of reduced levels of belonging (Hagerty \& Williams, 1999).

\section{Cognitive Burden and Depressive Symptoms}

Although cognitive impairment is evident after TBI, the relationship between depressed mood and cognitive function remains unclear. Montgomery (1995) described a reciprocal relationship between cognitive dysfunction and mood, but Satz and associates (1998) and McCleary and colleagues (1998) were unable to establish which neuropsychological 
correlates distinguished depressed from nondepressed 1st-year survivors.

Others have claimed that depressed mood after TBI contributes to diminished cognitive performance (AtteberryBennett, Barth, Loyd, \& Lawrence, 1986; MacNiven \& Finlayson, 1993) and study participants with significant clinical depression have had cognitive impairments comparable to those with brain injury (Brand, Jolles, \& Gispen-de Wied, 1992; Burt, Zembar, \& Niederehe, 1995; Cassens, Wolfe, \& Zola, 1990; Veiel, 1997). Williams and associates (2000) demonstrated a relationship between stress, depression, and cognitive performance among communitydwelling people with clinical depression. Furthermore, people with depressed mood reported delayed informationprocessing times (Brand \& Jolles, 1987; Hagerty, Williams, \& Liken, 1997) and significantly greater cognitive difficulties than did those without depression. Further study of specific neuropsychological correlates of depressed mood is needed because survivors have difficulties with attention and shortterm memory, which are cognitive functions necessary for effective community living.

Thus, with this cross-sectional survey design, the following hypotheses were tested:

Hypothesis 1. Postinjury chronic stress and post-TBI depressive symptoms are positively related.

Hypothesis 2. Interpersonal relatedness (postinjury sense of belonging and social support) and post-TBI depressive symptoms are inversely related.

Hypothesis 3. Cognitive burden-processing speed and accuracy - and post-TBI depressive symptoms are positively related.

\section{Methods}

Data were collected from 75 TBI survivors who sustained mild or moderate brain injury, were hospitalized for the injury, and were evaluated by a neuropsychologist with expertise in assessment of brain injury. All survivors were within 2 years of the injury and gave informed consent to participate in the study. Eligible participants were English-speaking survivors between 18-65 years old who were not psychotic at the time of their neuropsychological evaluation, had no preinjury neurological impairment, and had a relative or significant other $(\mathrm{R} / \mathrm{SO})$ who agreed to participate. Those who sustained severe injuries were excluded because of the likelihood that impaired cognitive function early after injury would make results on the cognitive battery of tests difficult to interpret.

Institutional review board approval was obtained from five brain-injury clinics affiliated with large urban teaching centers with specialties in trauma care. Former program participants and admitted survivors who met the eligibility criteria were invited by letter to participate. Data collection occurred over 15 months and ceased when 75 TBI participants were enrolled.

Participants signed informed consent forms and helped select the location, date, and time for data collection. Although the estimated time for data collection would be a single 90-minute testing period, accommodations were made for participant fatigue, scheduling conflicts, and transportation difficulties. The sequence of testing was consistent for all participants; the cognitive battery of tests was completed first, then measures of depression and postinjury stress, followed by interpersonal relatedness measures and demographic information. Data were collected in the presence of the principal investigator. Each participant was paid \$10. Both the participant and a family member were asked questions about severity of brain injury to ensure that retrospective data about the duration of unconsciousness and length of posttraumatic amnesia (PTA) were accurate. Data from survivors and family members were used only when they were consistent with chart data.

Estimates of brain injury severity. Traditional measures of brain injury severity were used: PTA, Glasgow Coma Scale (GCS) score, and duration of unconsciousness. PTA was defined as the duration in days of the survivors' ability to form continuous basic memories once out of coma. An instrument developed by van der Naalt, van Zomeren, Sluiter, and Minderhoud (1999) was used prospectively by nurses was used as a guide for family members to estimate the duration of PTA. GCS scores were obtained from emergency department data and only those who received a GCS score $>8$ were eligible for the study. However, if the admitting score was 3 and the next documented score was $>8$, the survivor was eligible for participation. The duration of loss of consciousness (LOC) was obtained by retrospective chart review.

Characteristics of mild brain injury were consistent with the definition established by the 1993 American Congress of Rehabilitation Medicine: PTA $<24$ hours and LOC $<30$ minutes. People with moderate brain injury had a duration of LOC exceeding 30 minutes but less than 2 weeks, a GCS score of $9-12$ or $13-15$ with an abnormal computed tomography $(\mathrm{CT})$ report.

Depressive symptoms. Two measures of depressive symptoms were used, the Center for Epidemiological Studies Depression Scale (CES-D) and the Neurobehavioral Functioning Inventory (NFI) depression subscale (D). On the CES-D, respondents rate the number of days (0-7) in the past week they experienced depressive symptoms. Higher scores denote greater levels of depression. This measure of depressive symptoms, never reported on with the TBI population, has excellent psychometric properties when used with the general public and has guidelines for severity of depressive symptoms (Devins \& Orne, 1985; Radloff, 1977). Convergent validity with the Hamilton Rating Scale (HRS), frequently used with the TBI population, was ( $r=.50$ to .80$)$. In this study, scores on the CES-D ranged from 0-57 $(M=20.45, S D=13.24)$ and the Cronbach's alpha coefficient was .78 .

The Neurobehavioral Functioning Inventory is a 76-item self-report inventory indicating problems currently experienced by people with neurological injury. It was designed for and validated with a sample of 520 Englishspeaking TBI survivors and their families, hence two versions were developed. Depressive symptoms, one of the six subscales (Kreutzer, Marwitz, Seel, \& Serio, 1996), showed construct 
validity with the Minnesota Multiphasic Personality Inventory (MMPI) depression scale $(r=.47)$. The mean depression score reported in the NFI manual was 33 for those with less than 14 days of unconsciousness. In this study, the Cronbach's alpha coefficient for depressive symptoms was $.92(X=30.69$, $S D=10.66)$. No attempt was made to quantify the onset and duration of depressive symptoms.

Postinjury stress. The Perceived Stress Scale, a 14-item Likerttype measure (Cohen, Kamarck, \& Mermelstein, 1983) was administered to determine participants' perceptions of chronic stress encountered over the previous 30 days. This measure correlates with the CES-D and Beck Depression Inventory (BDI) $(r=.70)$ and has good reliability (alpha=.85; Cohen et al., 1983; Cohen et al., 1995). Williams and associates (2000) reported the average perceived stress score for those with depression (measured with the BDI) and living in the community was 33.76. For this investigation, the range was 5-52 $(M=28.36$, $S D=9.45$ ) and alpha coefficient was .87.

Interpersonal relatedness was measured with two instruments, the Interpersonal Relationship Inventory (IRI), Tilden's 39-item, multidimensional measure of interpersonal relationships (Tilden, Nelson, \& May, 1990) and Hagerty's Sense of Belonging Instrument-Psychological (Hagerty \& Patusky, 1995). The IRI, derived from social exchange theory, contains three subscales ranked from 1-5 on a Likert scale. It has been used with a wide variety of adult populations: cancer patients, battered women, adult women in the community, and people with clinical depression (Hagerty \& Williams, 1999; Tilden et al., 1990). Hagerty and associates (1996) reported Cronbach's alpha coefficients of .93 for the social support subscale $(M=54.38)$ when tested with community-dwelling depressed men and women. For this investigation, the Cronbach's alpha for social support was $.88(M=51.07, S D=8.90$, range $=20-64)$.

Sense of belonging indicates fit and involvement with others, an object, or the environment. On this 18-item questionnaire, respondents rated their sense of connection to others on a 1-4 Likert scale; lower scores indicated low levels of belonging. When administered to communitydwelling college students, Roman Catholic nuns, and people with clinical depression and depressive symptoms, Cronbach's alpha ranged between .91-.96 (Brooks, 1999; Hagerty \& Patusky, 1995; Hagerty \& Williams, 1999). Hagerty and Patusky (1995) reported a mean score of $43.49(S D=10.54)$ when administered to depressed patients. For this investigation, the Cronbach's alpha coefficient was .95 and the range was 24-72 $(M=55.11, S D=11.8)$.

Six measures of directed attention and short-term memory were included in the cognitive battery (Williams et al., 2000): Letter and Star Cancellation, Necker Cube Pattern Control Test, Block Tapping, Faces, Balancing the Checkbook, and Delayed Recognition Word Span (DRWS).

Before beginning the cognitive battery, each participant was asked to rate conditions in the testing environment (lighting, noise, and temperature) and their perceived skill in using a computer. Then, written and verbal instructions for each test were given with a hands-on practice session. An investigator remained with the participant during the entire test to respond to technical questions. Scoring results for each test were saved in a comma-delimited-file for efficient merging with Statistical Package for the Social Sciences software (Windows 9.0 version). For each test, an accuracy score and a time-tocomplete score were obtained, and the total time to complete the cognitive battery was calculated for each participant.

\section{Results}

Demographic profile. This sample was predominately $(91.7 \%)$ Caucasian. Thirty-six $(48 \%)$ were women and 39 $(52 \%)$ were men. Nearly $91 \%$ were employed before the injury. The average age was 37.4 years $(S D=12.10$, range 19-60). Thirty-six percent were never married, $40 \%$ were married, and $20 \%$ were divorced, separated, or widowed. Four percent were living with a significant other. One third had annual household incomes over $\$ 75,000$ and $14.7 \%$ had annual incomes less than $\$ 15,000$.

Thirteen percent had been previously treated for alcohol or drug problems. Sixty-five percent rated their current health status as good to excellent. Forty-one percent were taking prescribed antidepressants, but no data were collected on the drug, dosage, and duration. Nearly $7 \%$ reported not having filled prescriptions for antidepressants. Twenty percent reported preinjury psychiatric disorders, such as anxiety or depression, $8 \%$ said they had suicidal thoughts the year before the injury, and $16 \%$ had a previous head injury.

Head injury severity. On the date of testing, 30 survivors were within 7 months of their injury, and 45 were between 7-25 months from the date of injury. Equal numbers of survivors had mild and moderate levels of injury. The average PTA duration was 7.65 days $(S D=8.92$, range $0-45$ days). Slightly more than $48 \%$ had abnormal CT results, showing small hemorrhages, hematomas, or contusions. The exact location of injury was not recorded. Nearly $71 \%$ reported that a motor vehicle crash was the mechanism of injury and $64 \%$ reported the duration of unconsciousness to be brief to less than an hour.

Measures of depressive symptoms. The NFI-D and CES-D were strongly correlated $(r=.85, p<.00$, one-tailed). Using published CES-D norms (Radloff, 1977), 36\% of this sample had scores not in the depression range and $20 \%$ had CES-D scores $>30.5$. Mean depression scores on the CES-D and NFI$\mathrm{D}$ did not differ significantly by age, sex, preinjury psychiatric diagnosis or suicide attempts, mild versus moderate level of injury, or previous TBI. Participants taking antidepressants had significantly higher mean levels of depression on the CES$\mathrm{D}$ and NFI-D ( $t=2.92, p=.005$ and $t=3.05, p=.003)$. Because the NFI-D was validated with TBI samples, this was the outcome variable for the following analyses.

Hypothesis 1. Examination of the relationship between postinjury stress and depressive symptoms was revealing. Postinjury stress alone helped explain $54 \%$ of the variance $\left[R^{2}=.55\right.$; Adjusted $\left.R^{2}=.54, F=87.72(1,73), p=.00\right]$. In addition, a significant and positive linear relationship was found between 
time since injury, postinjury stress, and depressive symptoms $\left[R^{2}=.64\right.$, Adjusted $\left.R^{2}=.63, F=63.73(2,72), p=.00\right]$.

Hypothesis 2. Postinjury sense of belonging was inversely related to depressive symptoms and explained $54 \%$ of the variance in post-TBI depressive symptoms, $F=87.72,(1,73)$, $p=.00$. When time since injury was added to the model, $58 \%$ of the variance was explained, $F=52.23,(2,72), p=.00$. These variables were unaffected by age, gender, or severity of injury.

Social support was correlated with depressive symptoms and was a significant explanatory variable in a simple regression model, $R^{2}=.17(1,73), F=15.43, p=.00$. Yet, when time since injury and sense of belonging were entered with social support (See Table), it was no longer significant.

Hypothesis 3. Scores on the cognitive battery of tests of directed attention and short-term memory did not explain significant variations in depression. Depressive symptoms also were not explained by the time to complete these tests, and the error rate or time to complete the battery did not correlate with head injury severity measures, perceived stress, or either measure of depressive symptoms. Still, the combination of the visual recognition portion and typed portion on the DRWS test yielded some significant findings. When these two tests of short-term memory were summed, a significant explanatory relationship was found between this score and depressive symptoms, $R^{2}=.19$, Adjusted $R^{2}=.17, F=8.60$ (2, $72), p=.00$. When these variables were added to the model, this relationship was largely unaffected by age or severity of injury. Yet, when this summed cognitive measure was included with the PSS and time since injury, the summed test no longer helped explain post-TBI depressive symptoms.

\begin{tabular}{|c|c|c|c|c|c|}
\hline \multicolumn{6}{|c|}{$\begin{array}{l}\text { Table. Regression of Depressive Symptoms on Postinjury } \\
\text { Sense of Belonging, Social Support, Perceived Stress, and } \\
\text { Time Since Injury }{ }^{\mathrm{a}}\end{array}$} \\
\hline & \multicolumn{2}{|c|}{$\begin{array}{c}\text { Unstandardized } \\
\text { coefficients }\end{array}$} & \multicolumn{2}{|c|}{$\begin{array}{l}\text { Standardized } \\
\text { coefficients }\end{array}$} & \\
\hline & $\beta$ & Std error & $\beta$ & $t$ test & $p$ \\
\hline Postinjury sense of belonging & -.37 & .08 & -.41 & 4.5 & .00 \\
\hline Social support & .002 & .09 & .02 & .31 & .76 \\
\hline Postinjury stress & .53 & .09 & .47 & 6.16 & .00 \\
\hline Time since injury & .40 & .11 & .24 & 3.75 & .00 \\
\hline
\end{tabular}

\section{Discussion}

Findings from research about clinical depression are extended to TBI survivors resulting in a beginning framework for explaining post-TBI depression. Perceived postinjury stress was a strong explanatory variable of post-TBI depression. Variability in depressive symptoms could also have been related to pain and the severity of brain injury, yet these findings resulted from regression analysis using more than three variables. To achieve a medium effect with regression analysis, power analysis showed that a minimum sample size of 80 is necessary for regression analysis with four independent variables (Cohen,
1992). Thus, a larger sample is required to better discern relationships among mediating factors of postinjury stress. Furthermore, longitudinal studies are needed with a nonbrain-injured control group.

Psychosocial variables, specifically postinjury sense of belonging and perceived stress showed a significant effect on post-TBI depression. Sense of belonging, but not social support, was a significant explanatory variable of post-TBI depression. This finding is consistent with studies of clinically depressed community samples (Hagerty \& Williams, 1999; Hagerty, Williams, Coyne, \& Early, 1996). Further, participants with mild and moderate levels of injury also reported psychosocial difficulties after TBI; previous studies of social relationships included mostly participants with severe levels of TBI. Despite a modest correlation between postinjury stress and sense of belonging $(r=-.60)$, they are distinct variables with acceptable tolerance statistics (.64 and .61 respectively) and variance inflation factors (1.56 and 1.63 respectively). Still, relationships among postinjury stress, sense of belonging, and depressive symptoms would become clearer with longitudinal study.

Although measures of short-term memory or attention did not correlate with any measure of brain injury severity, depression, or postinjury stress, participants who rated themselves depressed perceived their cognitive performance to be worse than did those without depression. This finding is consistent with findings by Satz and associates (1998) that people with depressed mood had more negative self-cognitions. The inability to account for survivor use of learned memory strategies may confound the findings regarding the computerized battery. Channon and Green (1999) suggested that people with depressed mood had differences in flexible use of memory strategies and limited motivation to incorporate them in testing.

\section{Conclusions}

This study indicated specific variables related to post-TBI depression, and it is a beginning framework for future study. Perceived postinjury stress and sense of belonging help explain nearly $75 \%$ of the variance in depressive symptoms and both might be amenable to interventions by nurses involved in community services for people recovering from TBI. Assisting survivors to identify and manage stressors associated with the recovery process and helping them fortify their social skills for community re-entry are indicated. Studies are needed with use of larger samples to determine how depression, stress, and interpersonal relatedness change during the recovery and re-integration process. Then, further examination of mediating variables, such as brain injury symptoms and pain, is required along with comparison of those findings in people with and without brain injury.

\section{References}

American Congress of Rehabilitation Medicine. (1993). Definition of mild traumatic brain injury. Journal of Head Injury Rehabilitation, 8, 86-87. 
Atteberry-Bennett, J., Barth, J.T., Loyd, B., \& Lawrence, E. (1986). The relationship between behavioral and cognitive deficits, demographics and depression in patients with minor head injuries. International Journal of Clinical Neuropsychology, 8, 114-117.

Baum, A., Gatchel, R., \& Schaeffer, M. (1983). Emotional, behavioral, and physiological effects of chronic stress at Three Mile Island. Journal of Consulting and Psychology, 51(34), 565572.

Bay, E. (2000). The biobehavioral correlates of post-traumatic brain injury depression. Journal of Neuroscience Nursing, 32(3), 169176.

Brand, A.N., Jolles, J., \& Gispen-de Wied, C. (1992). Recall and recognition memory deficits in depression. Journal of Affective Disorders, 25, 77-86.

Brand, N., \& Jolles, J. (1987). Information processing in depression and anxiety. Psychological Medicine, 17, 145-153.

Breslau, N., \& Davis, G. (1986). Chronic stress and major depression. Archives of General Psychiatry, 43(4), 309-314.

Brooks, G. (1999). Stress, depressive symptoms, interpersonal relatedness and HPA-Axis functioning in women. Unpublished doctoral dissertation, University of Michigan, Ann Arbor.

Burt, D., Zembar, M.J., \& Niederehe, G. (1995). Depression and memory impairment: a meta-analysis of its pattern, and specificity. Psychological Bulletin, 117(2), 285-305.

Busch, C., \& Alpern, H. (1998). Depression after mild traumatic brain injury: a review of current research. Neuropsychology Review, 8(2), 95-108.

Cassens, G., Wolfe, L., \& Zola, M. (1990). The neuropsychology of depression. Journal of Neuropsychiatry, 2(2), 202-213.

Channon, S., \& Green P. (1999). Executive function in depression: The role of performance strategies. Journal of Neurology, Neurosurgery and Psychiatry, 66, 162-171.

Cohen, J. (1992). A Power Primer. Psychological Bulletin, 112(1), 155-159.

Cohen, S., Kamarck, T., \& Mermelstein, R. (1983). A global measure of perceived stress. Journal of Health and Social Behavior, 24, 385-396.

Cohen, S., Kessler, R., \& Gordon, L. (Eds.). (1995). Measuring stress: a guide for health and social scientists (1st ed.; Vol. 1). New York: Oxford University Press.

Cohen, S., \& Wills, T. (1985). Stress, social support, and the buffering hypothesis. Psychological Bulletin, 98(2), 310-357.

Devins, G.M., \& Orne, C.M. (1985). Center for Epidemiologic Studies Depression Scale. In D.J. Keyser \& R.C. Sweetland (Eds.), Test critiques (pp. 144-159). Kansas City, MO: Test Corp of America.

Hagerty, B., Lynch-Sauer, J., Patusky, K., Bouwsema, M., \& Collier, P. (1992). Sense of belonging: A vital mental health concept. Archives of Psychiatric Nursing, 6(3), 172-177.

Hagerty, B., \& Patusky, K. (1995). Developing a measure of sense of belonging. Nursing Research, 44(1), 9-13.

Hagerty, B.M., \& Williams, R.A. (1999). The effects of sense of belonging, social support, conflict, and loneliness on depression. Nursing Research, 48(4), 215-219.

Hagerty, B., Williams, R. A., Coyne, J., \& Early, M. (1996). Sense of belonging and social and psychological functioning. Archives of Psychiatric Nursing, 10(4), 235-244.

Hagerty, B.M., Williams, R.A., \& Liken, S. (1997). Exploration of prodromal symptoms of major depression. American Journal of Orthopsychiatry, 67(2), 308-314.

Hibbard, M., Uysal, S., Kepler, K., Bogdany, J., \& Silver, J. (1998). Axis 1 Psychopathology in individuals with traumatic brain injury. Journal of Head Injury Rehabilitation, 13(4), 24-39.

House, J.S., Landis, K.R., \& Umberson, D. (1988). Social relationships and health. Science, 241, 540-545.

Jorge, R., Robinson, R., Arndt, S., Starkstein, S., Forrester, A., \& Geisler, F. (1993). Depression following traumatic brain injury: A 1 year longitudinal study. Journal of Affective Disorders, 27, 233-243.

Kendall, E., \& Terry, D.J. (1996). Psychosocial adjustment following closed head injury: A model for understanding individual differences and predicting outcomes. Neuropsychological Rehabilitation, 6(2), 101-132.
Kinsella, G., Moran, C., Ford, B., \& Ponsford, J. (1988). Emotional disorder and its assessment within the severe head injured population. Psychological Medicine, 18, 57-63.

Kozloff, R. (1987). Networks of social support and the outcome from severe head injury. Journal of Head Trauma Rehabilitation, 2(3), 14-23.

Kreutzer, J., Marwitz, J., Seel, R., \& Serio, C. (1996). Validation of a neurobehavioral functioning inventory for adults with traumatic brain injury. Archives of Physical Medical Rehabilitation, 77(2), 116-124.

Leach, L.R., Frank, R.G., Bouman, D.E., \& Farmer, J. (1994). Family functioning, social support and depression after TBI. Brain Injury, 8(7), 599-606.

Lezak, M. (1987). Relationships between personality disorders, social disturbances, and physical disability after traumatic brain injury. Journal of Head Injury Rehabilitation, 3, 57-69.

MacNiven, E., \& Finlayson, M.A.J. (1993). The interplay between emotional and cognitive recovery after closed head injury. Brain Injury, 7(3), 241-246.

Martelli, M., Zasler, N., \& MacMillan, P. (1998). Mediating the relationship between injury, impairment and disability: A vulnerability, stress and coping model of adaptation following brain injury. NeuroRehabilitation, 11, 51-66.

McCleary, C., Satz, P., Forney, D., Light, R., Zaucha, K., Asarnow, R., et al. (1998). Depression after traumatic brain injury as a function of Glasgow Outcome Score. Journal of Clinical and Experimental Neuropsychology, 20(2), 270-279.

Montgomery, G.K. (1995). A multi-factor account of disability after brain injury: implications for neuropsychological counseling. Brain Injury, 9(5), 453-469.

Moore, A., \& Stambrook, M. (1995). Cognitive moderators of outcome following traumatic brain injury: A model and implications for rehabilitation. Brain Injury, 9(2), 109-130.

National Institutes of Health. (1998). Rehabilitation of persons with traumatic brain injury. Paper presented at the NIH consensus statement, Washington, D.C.

Oddy, M., \& Humphrey, M. (1980). Social recovery during the year following severe head injury. Journal of Neurology, Neurosurgery, and Psychiatry, 43, 798-802.

Ownsworth, T., \& Oei, T. (1998). Depression after traumatic brain injury: conceptualization and treatment considerations. Brain Injury, 12(9), 735-751.

Radloff, L. (1977). The CES-D Scale: a self-report depression scale for research in the general population. Applied Psychological Measurement, 1(3), 385-401.

Rosenthal, M., Christensen, B., \& Ross, T. (1998). Depression following traumatic brain injury. Archives of Physical Medicine Rehabilitation, 79, 90-103.

Satz, P., Forney, D., Zaucha, K., Asarnow, R., Light, R., McCleary, C., et al. (1998). Depression, cognition, and functional correlates of recovery outcome after traumatic brain injury. Brain Injury, 12(7), 537-553.

Thomsen, I.V. (1984). Late outcome of very severe blunt head trauma: A 10-15 year second follow-up. Journal of Neurology, Neurosurgery, and Psychiatry, 47, 260-268.

Tilden, V., Nelson, C., \& May, B. (1990). The IPR Inventory: development and psychometric characteristics. Nursing Research, 39(6), 337-343.

van der Naalt, J., van Zomeren, A.H., Sluiter, W.J., \& Minderhoud, J.M. (1999). One year outcome in mild to moderate head injury: Acute injury characteristics related to complaints and return to work. Journal of Neurology, Neurosurgery, Psychiatry, 66, 207-213.

Veiel, H. (1997). A preliminary profile of neuropsychological deficits associated with major depression. Journal of Clinical and Experimental Neuropsychology, 19(4), 587-603.

Wells, K., Stewart, A., Hays, R., Burnam, A., Rogers, W., Daniels, M., et al. (1989). The functioning and well-being of depressed patients. JAMA, 262(7), 914-919.

Williams, R.A., Hagerty, B.M., Cimprich, B., Therrien, B., Bay, E., \& Oe, H. (2000). Changes in directed attention and short-term memory in depression. Journal of Psychiatric Research, 34, 227-238. 